\title{
Emotion Appraisal Tendencies and Carryover: How, Why, and ... Therefore?
}

\author{
J. Frank Yates \\ The University of Michigan
}

\begin{abstract}
The effects and ideas described by Han, Lerner, and Keltner (2007) are fascinating and important. Rightly so, they are likely to capture researchers' and practitioners' attention for some time. This commentary offers several questions (and speculations as to possible answers) that hopefully will be the focus of some of that attention. One theoretical question concerns the mechanisms whereby emotional carryover effects occur and the purposes that such effects might serve. A practical one is about the prescriptive implications of carryover effects and appraisal tendency influences more generally, particularly in concrete, real-life decision situations.
\end{abstract}

Han, Lerner, and Keltner (2007) discuss a host of intriguing phenomena and ideas, but the facts and proposals do more than just fascinate. They are likely to have significant and lasting impact on judgment and decision scholarship generally as well as in the specialized domain of consumer behavior. Because the findings are often so surprising, they will force us to work especially hard to make sense of them and, therefore, deepen significantly our understanding of how people decide fundamentally. As is always the case in instances like this, that work will be directed toward answering fairly specific questions inspired by Han et al.'s analysis. These remarks pose several questions that are at the top of my personal list. In some cases, I simply ask the questions. In others, though, I cannot resist offering what seem to me to be some plausible potential answers, too. Hopefully, my curiosity will be satisfied by future studies if the questions cannot be answered (perhaps by Han et al.) on the basis of work already in the literature.

\section{THE APPRAISAL-TENDENCY FRAMEWORK- CONCRETELY}

The Appraisal-Tendency Framework (ATF), as described by Han et al. (2007) and in the literature more generally, is an undeniably compelling and useful set of ideas. Nevertheless, its concepts and propositions are framed in terms that

Correspondence should be addressed to J. Frank Yates, Psychology Department and Ross School of Business. The University of Michigan. Ann Arbor, MI 48109. E-mail: jfyates@umich.edu are unusually abstract and hard to pin down, by the customs of judgment and decision-making psychology and marketing research. Among other things, this limits the testability and application of the framework. For instance, it is unclear what, exactly, is being appraised when the framework speaks of "appraisal tendencies." Thus, my first question is simple and uninteresting but essential, it seems: What, concretely, are the various constructs of the framework, and how can we recognize their manifestations in real "decision life?" In what follows, I briefly sketch specific decisionmaking interpretations that seem consistent with what has been written in the ATF literature, but are my interpretations really consistent with the original intent?

Figure 1 sketches a possible instantiation of the ATF in the context of a decision-making episode. As always (cf. Yates, 2003), the episode starts with the acknowledgment that a problematic situation exists (or is impending)-for example, that one's job is threatened. Per the ATF, that leads to an emotion or an appraisal of the situation that is in a recursive, mutually reinforcing relation with that emotion-that is, the emotion (e.g., fear) might come first, then lead to a particular kind of appraisal of the situation (e.g., that it is a "fear-type" situation), or vice versa (e.g., there is first an appraisal that the situation is of the fear type and this, therefore, evokes a sense of fear). Then two alternative paths are possible. On the "logical path," the emotion and the appraisal predispose the person to choose and pursue a particular action that is directed toward solving the problem in question (e.g., saving one's job or gaining a new one in certain ways). If the situation is indeed resolved satisfactorily (i.e., the decision succeeds), the situation has changed; therefore, naturally, so does its appraisal and the 


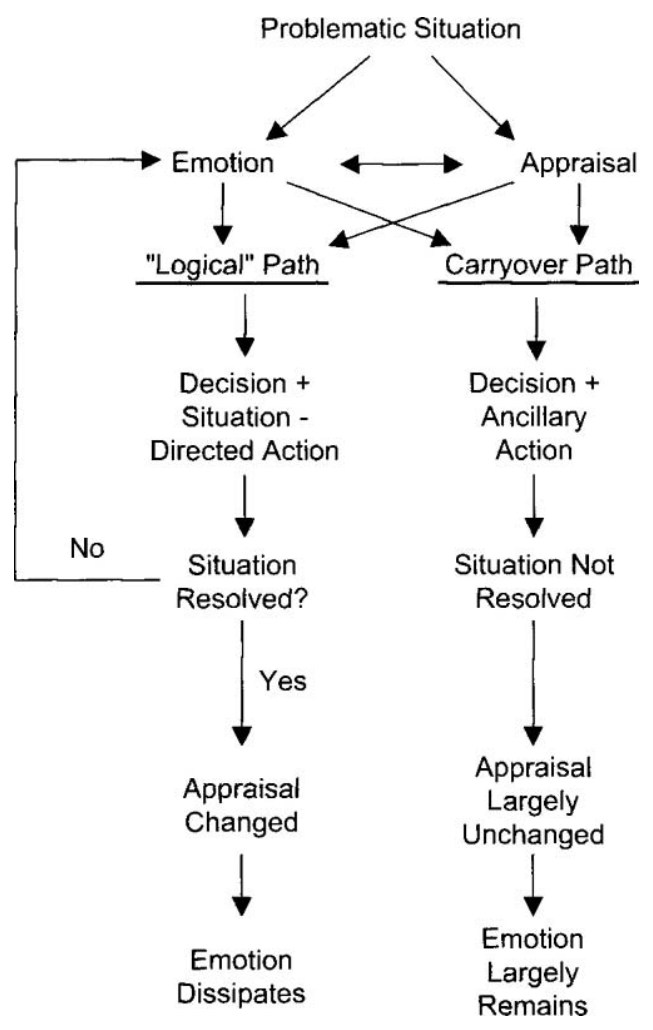

FIGURE 1 A possible interpretation of the Appraisal-Tendency Framework in a decision-making context.

corresponding emotion. If the decision fails, then none of that happens and, presumably, the persisting emotion and appraisal continue to encourage and facilitate "matching" kinds of actions (e.g., one is consistent with fear of the source of one's threatened job loss). The "carryover path" is very different and a novel element of the ATF. There, the emotion and appraisal encourage and facilitate similar kinds of actions but those that are "ancillary" in the sense that they have no bearing on the situation that launched the decision episode in the first place (e.g., the person made fearful for his job proceeds with unusual caution in non-job related social settings). Of course, this does nothing to resolve that situation, and the original emotion and appraisal largely remain, as implied by Han et al. (2007). I use the qualifier largely because the reviewed evidence is ambiguous on the point. Although, as suggested later, one can make a case that there will be some ameliorating effect of pursuing the carryover path.

In a decision situation, what does the decision maker sensibly "appraise?": any aspect of the situation that bears on the wisdom of actions one might take to solve the problem in question (e.g., the formidability of the adversaries or competitors threatening one's job or the prospects of finding new employment). The ATF, building on the formulation of Smith and Ellsworth (1985), singles out several specific "appraisal dimensions" for special consideration: certainty, pleasantness, attention, control, anticipated effort, and responsibility, mainly because there is evidence that those dimensions influence and distinguish various emotions. However, it would be useful to concretize these dimensions (as well as the myriad others that undoubtedly would matter) as they are realized in decision problems. Thus, for the ATF to be most valuable, we would need to have answers to questions such as, "Responsibility for what, as it bears on the decision problem at hand?" (The answer, for instance, might be who is responsible for a threat to one's livelihood, to inform possible appeals or countermeasures.)

A plausible interpretation of an ATF "appraisal" in a decision context is the decision maker's conclusion as to the state of a particular germane aspect of the problematic situation. In one situation, for instance, the decider might conclude that a specific competitor, "Jane," is responsible for the threat to his job. In another, he might conclude that nebulous "economic conditions" bear the blame. The implications for what makes sense to do about the job problem would be radically different. We might envision a profile of state assessments for all the pertinent situation aspects, representable generically as in Figure 2 a. A core concept of the ATF is that of "appraisal tendencies." In this conjectured decision interpretation, an appraisal tendency would be a distinctive pattern of aspect assessments that is especially likely to be observed. The ATF asserts that particular emotions are associated with particular tendencies. Substantively, this would mean that those emotions are associated with dispositions to expect or come to certain characteristic conclusions about the aspects in question. Figure $2 \mathrm{~b}$ illustrates the idea. The suggestion is
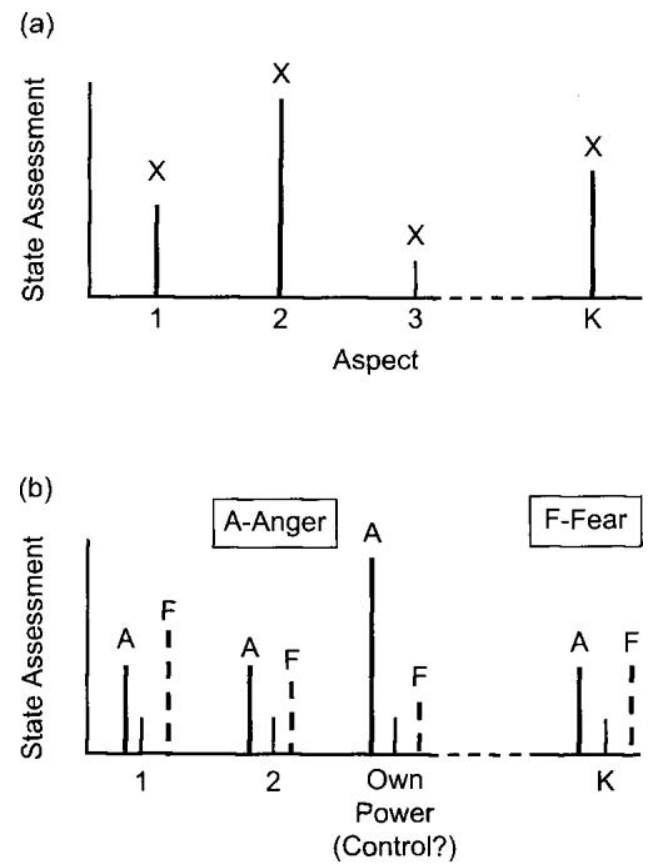

Aspect

FIGURE 2 Possible representations of state assessment profiles: (a) generically and $(b)$ in contexts of anger $(A)$ and fear $(F)$. 
that anger and fear have different appraisal profiles. The ATF asserts, specifically, that the appraisal tendencies for control differ greatly for anger and fear. In a given decision situation, "control" would have to have a particular manifestation. As suggested in the figure, in many situations, it seems intuitive that that manifestation would entail the power of the decision maker relative to that of the persons or forces that must be overcome to resolve or control the situation at hand. After all, we "fear" opposition that is more powerful than us and which therefore can harm us, but we become "angry" with weaker opposition that we can overwhelm. More importantly, consistent with the ATF, fear predisposes us for retreat and facilitates that action. In contrast, anger predisposes us for attack and amplifies its ferocity.

So, the challenge remains: What are the constructs and claims of the ATF for decision problems - concretely? Are the suggestions presented here close, or are they entirely different?

\section{UNDERLYING MECHANISMS}

ATF research has provided convincing evidence of numerous striking and surprising effects. However, it remains a mystery how and why some of those effects occur (i.e., what the underlying mechanisms are). As Lerner and Keltner (2001) put it, the ATF maintains that phenomena such as emotion-keyed appraisal tendencies are functional, being "tailored to help the individual respond to the event that evoked the emotion" (p. 146). Thus, extending the previous illustration, an appraisal that anger is appropriate for a given situation would entail an assessment that the decision maker probably has the power to overcome the opposition that is present. Therefore, "attack" would not be a suicidal option to pursue, although it might well be fatal in circumstances giving rise to a fear appraisal. This functionality assumption heightens what, in my view, is the most compelling puzzle of all-carryover effects. Since they have no impact on the original source of the emotion (see Figure 1), the problem that grabbed the decision maker's attention and initiated the decision episode under way, why do carryover effects occur? Why, for instance, does the ambient or incidental anger a person brings with her into an investment situation induce her to make risk-seeking choices that can have no influence on whatever angered her in the first place? Answering questions like these arguably should be the first order of business for emotions research within the ATF franchise. Several plausible mechanisms suggest themselves-ones involving coherence striving, associative networks, and "emotional state management," which may or may not be entirely independent of one another.

\section{Coherence Striving}

Our situations in the world are often complex and incoherent (i.e., seemingly inconsistent facts coexist). For instance, people we like sometimes do things we think are wrong, even reprehensible; an apartment we might lease has a beautiful layout, but is located in a bad part of town. For decades, enormous amounts of research have directly and indirectly pointed to people's abhorrence of incoherence and, therefore, our tendency to reconstrue apparent facts to reduce the contradictions we perceive. The best known classic line of scholarship documenting such phenomena is identified with Festinger's (1962) cognitive dissonance ideas. However, there have been numerous other streams, including several important recent ones.

Slovic (1966) studied respondents' judgments of target persons' intelligence, based on a variety of cues such as scores on English effectiveness, high school grades, and study habits. He found that, in cases where two cues contradicted each other (e.g., high English score but weak high school grades), participants made judgments differently than when the cues tended to agree (e.g., high English score and strong grades). In the former case, respondents tended to underweight one of the conflicting items, in effect, achieving coherence by discounting inconvenient indications of inconsistency. More recently, Simon, Krawczyk, and Holyoak (2004) gave participants information early in the deliberation process that favored one alternative job offer rather than another, thereby inducing a tentative preference for that option. Simon et al. found that this colored the remainder of the deliberations such that participants reconstrued almost all considerations (e.g., how important office size was) such that those features supported the tentative preference too. Even closer to this domain, Finucane, Alhakami, Slovic, and Johnson (2000) reported evidence of an "affect heuristic" that appears to contribute to a most interesting phenomenon-the tendency for people to believe that if some endeavor (e.g., nuclear power) is beneficial, then it must also entail little risk, and vice versa, that highly risky enterprises offer minimal benefits. (Such beliefs seem at odds with reality in that, usually, the world tolerates risky activities only because they also promise substantial rewards that compensate for the high risk.) It is not implausible that the affect heuristic is a manifestation of coherence striving too. Imagine a person who has come to support nuclear power because of its benefits. To maintain a sense of coherence, that person might well come to construe the riskiness of nuclear power to be minimal also, reinforcing the impression of all-around worthiness of that technology.

What are the possible implications for carryover effects? Suppose that a particular emotion is being experienced-for example, fear rather than anger over the prospect of losing one's job. At that particular moment, fear dominates the person's perspective on the world. Then, in the service of maintaining coherence, many other things in that perspective should be seen (and felt) as warranting fear too. Therefore, seemingly justifiably, decisions on matters having nothing to do with the original genesis of the fear (e.g., 
choosing a car seat for one's child) would have the character of fear-driven choices as well (e.g., risk aversion).

\section{Associative Networks}

A second proposed set of mechanisms underlying carryover effects has, in effect, been in the literature for some time, being suggested by Forgas (1995) in his discussion of the affect infusion model. However, it still seems to be a viable candidate as a contributor to these effects. In essence, the proposal suggests that when a certain emotion is experienced, it activates particular nodes in the person's associative networks. This implies that associated facts come to mind or are recognized more readily than otherwise; therefore, conclusions consistent with those facts seem more defensible than if they had not been acknowledged. Significantly, as far as carryover effects are concerned, associative network activation proceeds very rapidly and independently of propositional knowledge or reasoning. Therefore, they can seemingly "upstage" other, perhaps more reasoned, considerations. Therefore, if a person is in a fearful state, this should activate fear-like elements of the associative networks of almost anything under consideration at the moment. Thus, the purchase of a child's car seat at that time would be dominated by those elements and would be unaffected by other factors that literally never enter the decisionmaker's mind. A recent study by Chua, Yates, and Shah (2006) is consistent with the role of associative networks in carryover-like phenomena. These investigators found evidence that certain types of graphical (as opposed to numerical) displays of risk information seem to have a rapid, attention-capturing impact on overall risk assessments and choices. Moreover, those effects appeared to, in part, be attached to entire decision alternatives, not just logically reasonable elements such as chances of harm (see Bechara, Damasio, Tranel, \& Damasio, 1997; Epstein, 1994.)

\section{Emotional State Management}

Considerable current scholarship in decision making centers on what might be called "emotional state management"people's efforts to, essentially, make themselves feel good emotionally, or at least not feel bad. Some of that work has focused on people's efforts to choose alternatives whose results make them feel good (e.g., Mellers, Schwartz, Ho, \& Ritov, 1997). However, other studies have emphasized the emotional states of decision makers while deciding. Studies framed in terms such as "mood maintenance," "mood repair," and even "mood enhancement," including those by Isen and Geva (1987), are representative. In a nutshell, these kinds of investigations demonstrate that conditions surrounding a decision-making episode affect the decider's emotional state. Further, the aim of making that state pleasant or at least tolerable partly dictates how and what the person decides (cf. Loewenstein, Weber, Hsee, \& Welch,
2001). This second variety of emotional state management conceptually is not unlike therapies for coping with clinical mood disorders or, more familiarly, what people do when they drink alcohol to forget their troubles or to pick up their spirits.

What is the relevance to carryover effects? Han et al. (2007) refer to each emotion's "motivational properties that fuel carryover" (p. 160). However, they do not elaborate on what they mean. Regardless, emotional state management considerations are a plausible driver of carryover effects that seem consistent with this idea. Recall Figure 1, and suppose that a problematic situation arises and evokes a certain emotion (e.g., anger). Per the ATF, the associated appraisal tendencies predispose the person toward particular kinds of decision alternatives that are promising in terms of successfully addressing the initiated decision episode (e.g., protecting one's job in the face of an anger-inducing threat)-that is, solving the original problem provides part of the decision maker's incentives. However, the evoked emotion adds more. It seems likely that, in many instances, the experience of the emotion in question (e.g., fear or anger) is inherently unpleasant in its own right; it makes the person feel bad. Further, the "expression" of that emotion in the form of actions consistent with the associated appraisal tendency might, in and of itself, relieve at least part of that bad feeling; this should occur regardless of the object of such actions (i.e., as suggested in Figure 1, ancillary carryover actions could indeed serve a compelling function). That would be why, for example, being mean to his children might, sadly, provide satisfaction to an angry worker worried about his job security.

\section{THEREFORE?: PRESCRIPTIONS}

Han et al. (2007) submit that the ATF has significant practical implications for consumer decision making, and they buttress this assertion with discussions of findings about risk assessments and the assessment of monetary value (e.g., people's willingness to pay certain amounts of money for particular goods). These findings are compelling, but I must confess to some skepticism about how readily consumers and marketers can translate ATF principles and findings into useful prescriptions. Surely, for instance, one should not advise marketers to purposely make consumers angry so that they would take greater risks in their purchases, right? So, what advice does make sense? This is an important challenge for future "translation" research. The precise shape that such efforts might take is unclear. However, my hunch is that one fruitful avenue would entail teaching people how to manage in the event that particular emotions just happen to arise naturally. For example, prescriptions for consumers might begin like so: "Be aware that your emotional state is likely to affect significantly what you choose to do, and those choices might not be in 
your long-term interests. Let me explain, and then let's consider how you can protect yourself." Advice to sellers might be prefaced this way: "If you notice that a customer is angry (or happy, sad, etc.), you might want to take into account what research suggests is likely to be the way that customer is seeing things. We can then consider how you should approach that person."

\section{ACKNOWLEDGMENT}

It is my pleasure to thank Andrea Angott, Lydia Chen, and Jennifer Harp for their useful comments on the issues addressed in this article.

\section{REFERENCES}

Bechara, A., Damasio, H., Tranel, D., \& Damasio, A. R. (1997). Deciding advantageously before knowing the advantageous strategy. Science, 275, 1293-1295.

Chua, H. F., Yates, J. F., \& Shah, P. (2006). Risk avoidance: Graphs vs. numbers. Memory \& Cognition, 34, 399-410.

Epstein, S. (1994). Integration of the cognitive and psychodynamic unconscious. American Psychologist, 49, 709-724.
Festinger, L. (1962). A theory of cognitive dissonance. Stanford, CA: Stanford University Press.

Finucane, M. L., Alhakami, A., Slovic, P., \& Johnson, S. M. (2000). The affect heuristic in judgments of risks and benefits. Journal of Behavioral Decision Making, 13, 1-17.

Forgas, J. P. (1995). Mood and judgment: The affect infusion model (AIM). Psychological Bulletin, 117, 39-66.

Han, S., Lerner, J. S., \& Keltner, D. (2007). Feelings and consumer decision making: The appraisal-tendency framework. Journal of Consumer Psychology, 17, 158-168.

Isen, A. M., \& Geva, N. (1987). The influence of positive affect on acceptable level of risk: The person with a large canoe has a large worry. Organizational Behavior and Human Decision Processes, 39 , 145-154.

Lerner, J. S., \& Keltner, D. (2001). Fear, anger, and risk. Journal of Personality and Social Psychology, 81, 146-159.

Loewenstein, G. F., Weber, E. U., Hsee, C. K., \& Welch, N. (2001). Risk as feelings. Psychological Bulletin, 127, 267-286.

Mellers, B. A., Schwartz, A., Ho, K., \& Ritov, 1. (1997). Decision affect theory: Emotional reactions to the outcomes of risky options. Psychological Science, 8, 423-429.

Simon, D., Krawczyk, D. C., \& Holyoak, K. J. (2004). Construction of preferences by constraint satisfaction. Psychological Science, 15, 331-336.

Slovic, P. (1966). Cue consistency and cue utilization in judgment. American Journal of Psychology, 79, 427-434.

Smith, C. A., \& Elsworth, P. C. (1985). Patterns of cognitive appraisal in emotion. Journal of Personality and Social Psychology, 48, 813-838.

Yates, J. F. (2003). Decision management. San Francisco: Jossey-Bass. 